\title{
Renaissance of Aspirin for the Primary and Secondary Prevention of Venous Thromboembolism
}

\author{
Allison E. Burnett • Linda R. Kelly • \\ Tuesdy Horner $\cdot$ Dukens LaBaze $\cdot$ \\ Brandi N. Bowman · Charles E. Mahan
}

Published online: 15 October 2013

(C) Springer Science+Business Media New York 2013

\begin{abstract}
Long-standing controversy has surrounded aspirin as an antithrombotic modality to prevent venous thromboembolism (VTE), particularly in the orthopedic surgery arena. New data are emerging on the use of aspirin for both primary VTE prevention and secondary prevention of recurrent VTE after an initial anticoagulation course. This review article will provide the history behind the controversy, information regarding recent or ongoing clinical trials of aspirin for primary VTE prevention in major orthopedic surgery, evidence for aspirin in secondary prevention of recurrent VTE, and suggestions for future research. Aspirin may be a reasonable option for VTE prevention in certain major orthopedic surgery patients. Additionally, investigation among non-orthopedic populations such as the acutely ill medical patient and the non-orthopedic surgery patient is encouraged to provide further safety and efficacy data.
\end{abstract}

\section{A. E. Burnett}

University of New Mexico College of Pharmacy, University of

New Mexico Hospital, Albuquerque, NM, USA

e-mail: aburnett@salud.unm.edu

L. R. Kelly · T. Horner · B. N. Bowman · C. E. Mahan ( $\square)$

Presbyterian Healthcare Services, University of New Mexico

College of Pharmacy, 1100 Central Ave. SE, Albuquerque,

NM 87106, USA

e-mail: cmahan@phs.org

L. R. Kelly

e-mail: 1kelly@phs.org

T. Horner

e-mail: thorner@phs.org

B. N. Bowman

e-mail: bbowman@salud.unm.edu

D. LaBaze

University of New Mexico School of Medicine, Albuquerque, NM, USA

e-mail: DLaBaze@salud.unm.edu
Keywords Aspirin · Venous thromboembolism · Anticoagulants · Antiplatelets · Prevention · Recurrent

$\begin{array}{ll}\text { Abbreviations } \\ \text { AAOS } & \text { American Academy of Orthopedic Surgeons } \\ \text { ACCP } & \text { American College of Chest Physicians } \\ \text { ATE } & \text { Arterial thromboembolism } \\ \text { CI } & \text { Confidence interval } \\ \text { CRNM } & \text { Clinically relevant non-major } \\ \text { DSMB } & \text { Data Safety and Monitoring Board } \\ \text { DVT } & \text { Deep vein thrombosis } \\ \text { GI } & \text { Gastrointestinal } \\ \text { HFS } & \text { Hip fracture surgery } \\ \text { HR } & \text { Hazard ratio } \\ \text { LMWH } & \text { Low-molecular-weight heparin } \\ \text { MI } & \text { Myocardial infarction } \\ \text { NJR } & \text { National Joint Registry } \\ \text { NVAF } & \text { Non-valvular atrial fibrillation } \\ \text { OMA } & \text { Oral monotarget anticoagulants } \\ \text { OR } & \text { Odds ratio } \\ \text { PE } & \text { Pulmonary embolism } \\ \text { SCIP } & \text { Surgical care improvement project } \\ \text { SD } & \text { Standard deviation } \\ \text { THR } & \text { Total hip replacement } \\ \text { TKR } & \text { Total knee replacement } \\ \text { UFH } & \text { Unfractionated heparin } \\ \text { VTE } & \text { Venous thromboembolism } \\ & \end{array}$

\section{Introduction}

Venous thromboembolism (VTE) is comprised of deep vein thrombosis (DVT) and pulmonary embolism (PE). It is estimated that up to 300,000 deaths annually in the USA 
are attributable to VTE [1]. Antithrombotics typically used for VTE prevention consist of the anticoagulants warfarin, unfractionated heparin (UFH), low-molecular-weight heparins (LMWHs), fondaparinux, oral monotarget anticoagulants (OMAs) such as dabigatran, rivaroxaban, and apixaban, and, at times, antiplatelets primarily including aspirin. Significant controversy has surrounded the use of aspirin for VTE prevention over the last three decades. Purported disadvantages predominantly relate to aspirin being less efficacious as compared to anticoagulants in reducing VTE and difficulties in assessing patient adherence. Aspirin possesses some attractive advantages over other prophylactic agents such oral administration, nonprescription status, low acquisition cost, and no need for routine monitoring (Table 1 ). As such, there is a recently rekindled interest in aspirin's use in various settings including primary VTE prevention [2••] as well as secondary prevention of VTE after an initial course of anticoagulation $[3 \cdot \bullet, 4 \bullet \cdot]$. The purpose of this review is to evaluate aspirin studies, both old and new, discuss clinical controversy surrounding aspirin in VTE prevention, describe the impact on national VTE prevention guidelines, and highlight suggested areas of future research.

\section{Traditional Pharmacologic Options for VTE Treatment and Prophylaxis}

Warfarin has been used as an oral anticoagulant in the USA since 1954. Aside from VTE prophylaxis in medical and surgical patients, it is the gold standard to which other anticoagulants used for VTE treatment are compared. Warfarin has a prolonged half-life of around 40 hour and a delayed onset and offset of action. Thus, peri-procedural "bridging" with a short-acting anticoagulant during initiation and/or interruption of therapy in high-risk patients is occasionally warranted. Warfarin dosing is affected by numerous drug-drug and drug-food interactions. Individualized dosing and routine monitoring are required, making warfarin a labor-intensive therapeutic option [5, 6].
Injectable agents, such as LMWHs, fondaparinux, and UFH, have been widely used for VTE prophylaxis in medical and surgical patients, including use after total hip replacement (THR), total knee replacement (TKR), and hip fracture surgery $\left(\mathrm{HFS} ;\left[\mathbf{7}^{\circ}\right]\right)$. They are often used as induction agents when transitioning a patient with acute VTE to warfarin or for peri-procedural bridging. In patients with malignancy and acute VTE, LMWHs are recommended as monotherapy for the first 3-6 months of treatment due to better efficacy, fewer interactions, and less risk of adverse events [8-10]. Importantly, in patients with severe renal impairment (i.e. creatinine clearance $<30 \mathrm{ml} /$ min), LMWHs should be used with caution, while fondaparinux is contraindicated [11].

OMAs have been introduced in recent years in an attempt to overcome some of the disadvantages of warfarin. OMAs have a more advantageous pharmacokinetic profile, do not require routine monitoring, and have fewer drug, disease, and dietary interactions than warfarin [6]. OMAs should also be used with caution in severe renal impairment due to varying renal clearances. In the USA, an oral direct Factor II inhibitor, dabigatran, and two oral direct Factor Xa inhibitors, rivaroxaban and apixaban, have been approved for stroke prevention in non-valvular atrial fibrillation (NVAF). To date, rivaroxaban is the only OMA FDA-approved for VTE prevention in THR/TKR and VTE treatment.

\section{The Role of Aspirin in VTE}

Long-standing controversy exists surrounding the use of aspirin to prevent VTE [12]. Opponents of aspirin have maintained that it may not provide adequate reductions in life-threatening thromboembolic events, particularly within the low-flow, low-shear venous system where fibrin and red blood cell-rich clots predominate. First patented by Bayer in 1889, aspirin has a long history of clinical use for multiple prothrombotic conditions [13]. It is currently recommended for stroke prevention in low-risk atrial fibrillation patients (e.g., CHADS = 1), certain valvular
Table 1 Comparison of agents used for VTE $[6,11,17,70]$

\begin{tabular}{llllll}
\hline & Onset & Offset & Route & Antidote & Acquisition cost \\
\hline Aspirin & Rapid & Slow & Oral & No & Very low \\
Warfarin & Slow & Slow & Oral/IV & Yes & Low \\
LMWH & Rapid & Rapid/intermediate & SubQ & Yes & High \\
Fondaparinux & Rapid & Slow & SubQ & No & High \\
UFH & Rapid & Rapid & SubQ & Yes & Low \\
Dabigatran & Rapid & Rapid/intermediate & Oral & No & High \\
Rivaroxaban & Rapid & Rapid/intermediate & Oral & No & High \\
Apixaban & Rapid & Rapid/intermediate & Oral & No & High \\
\hline
\end{tabular}


diseases, and secondary prevention of non-cardioembolic ischemic stroke [14-16]. Aspirin is also a mainstay of therapy in prevention of systemic arterial thromboembolism (ATE). There has been recent rejuvenation in the interest of using aspirin for primary and secondary prevention of venous events because of its non-prescription status, ease of use, and low cost compared to other antithrombotics.

Aspirin irreversibly inhibits the enzyme cyclooxygenase, which is responsible for thromboxane A2 and prostaglandin synthesis. This, in turn, impairs platelet adhesion, activation, and aggregation. Platelet inhibition effects last the lifespan of the platelet, approximately 5-9 days. Aspirin is metabolized via plasma esterases, as well as hepatic conjugation, and metabolites are eliminated in the urine. Because of the multiple routes of elimination, aspirin can be given to patients with renal or hepatic failure [17]. Side effects relate primarily to bleeding, gastrointestinal (GI) tract irritation, and rare hypersensitivity reactions. Most drug interactions are with agents that exacerbate GI irritation and/or bleeding effects such as other antiplatelet agents and non-steroidal anti-inflammatory agents [18]. While salicylate levels can be obtained, there is no specific antidote [19]. In some emergency situations, transfusion of functioning donor platelets can be considered with caution [20]. Overall, aspirin has been employed primarily for its antithrombotic effects within the high-pressure, high-shear arterial system where platelet-rich thrombi predominate. However, with recent advances in our understanding of how clot formation occurs, shifting from a "cascade" model to a "cell-based" model, it appears platelets have a key supporting role in the initiation, amplification, and propagation phases of VTE formation [21, 22]. Thus, there is biological rationale to support the premise of aspirin in prevention of VTE.

\section{Differences Between AAOS and ACCP Guidelines in the Orthopedic Population}

One of the most controversial areas over the last several decades has surrounded the use of aspirin in major orthopedic surgeries, specifically HFS, THR, and TKR. Annually, in the US, over 500,000 procedures for both THR and TKR are performed, with that number expected to grow by 175 and $675 \%$ by 2030, respectively [23]. THR patients are at higher risk for proximal DVT, whereas patients that undergo TKR have a higher incidence of distal DVT [24, 25]. Previous editions of American College of Chest Physicians (ACCP) guidelines specifically recommended against aspirin as monotherapy for VTE prevention in this population [26]. Interestingly, however, the more recent 2012 ACCP guidelines now recommend aspirin as an option for TKR, THR, and HFS (all grade 1B) as compared to no thromboprophylaxis $\left[7^{\bullet}, 27 \bullet\right]$. This change was primarily driven by a shift towards basing recommendations on the use of prophylaxis to reduce "patient-important" symptomatic VTE events only, rather than also including asymptomatic events, as well as taking into consideration the hazard of symptomatic bleeding (i.e. net clinical benefit $[12,26]$ ). These significant changes in recommendations have come under considerable scrutiny [28]. Importantly, while aspirin is a recommended ACCP option, LMWH is "suggested" as the preferred agent over other options including aspirin (grade 2C [7•]). The ACCP guidelines should be interpreted and applied cautiously, because findings of the PE prevention (PEP) trial (discussed below) have been extrapolated to both HFS and THR/TKR patients, even though no significant benefit was found in the PEP THR/TKR cohort.

Orthopedic surgeons tend to place a higher value on preventing bleeding events, whereas non-surgeons tend to place a higher value on preventing thrombotic events. As such, there has traditionally been strong opposition between the American Academy of Orthopedic Surgeons (AAOS) and the ACCP regarding VTE prevention. However, like the ACCP, the AAOS has also made some changes in their most recent guidelines [27•]. AAOS has changed the title of their document to "Preventing Venous Thromboembolic Disease in Patients Undergoing Elective Hip and Knee Arthroplasty" whereas previously, they focused only on prevention of symptomatic PE. The AAOS guideline, like the ACCP guideline, recommends pharmacologic prophylaxis for THR/TKR patients. However, they do not make recommendations for any specific prophylactic drug, with the exception of recommending aspirin for patients with higher bleeding risks. The AAOS does recommend mechanical compressive devices for patients with known bleeding disorders and/or hepatic disease. For patients with a history of previous VTE, they recommend a dual approach of pharmacologic agents and mechanical compression devices. The use of aspirin in this patient population is complicated by the Centers for Medicare and Medicaid's surgical care improvement project (SCIP), which affects hospital reimbursement. SCIP does not currently recognize aspirin as an appropriate sole prophylactic agent. However, aspirin can be used in a multimodal approach, along with mechanical devices, for patients who undergo elective THR/TKR or HFS and have contraindications to SCIP-recognized pharmacologic agents [12, 29]. Beginning January 2014, the use of aspirin alone for THR/TKR and HFS will be endorsed by SCIP (http:// www.qualitynet.org/dcs/ContentServer?c=Page\&pagename= QnetPublic\%2FPage\%2FQnetTier4\&cid=1228773265583, accessed 30 Sep 2013).

\section{Aspirin for Primary Prevention of Venous Thromboembolism (VTE)}

The efficacy of anticoagulants in reducing morbidity and mortality associated with VTE was proven nearly 50 years 
ago, and that efficacy has been bolstered by numerous clinical trials and meta-analyses over the years [30-35]. As a result, anticoagulants have become the preferred agents for primary prevention of VTE.

\section{The PEP Trial}

Early clinical trials examining aspirin for post-surgical VTE prevention had key methodological limitations, and meta-analyses generated conflicting results [36-42]. Consequently, aspirin was viewed by most as inferior to anticoagulants for primary VTE prevention. This collective body of low-quality, inconclusive evidence prompted the PEP trial, which was published in 2000 [43]. To date, this is the largest, randomized, placebo-controlled trial examining aspirin for VTE prevention. It included 13,356 patients undergoing HFS and another 4,088 patients undergoing elective THR or TKR. Patients were randomized to receive aspirin $160 \mathrm{mg}$ daily or placebo for 5 weeks. Approximately 35-44\% of patients received concomitant UFH or LMWH, and $30 \%$ had thromboembolicdeterrent stockings, making the true impact of aspirin on VTE difficult to discern. In the HFS cohort, aspirin use resulted in a $29 \%$ reduction in symptomatic DVT [1.0 vs. $1.5 \%$; hazard ratio (HR) $0.71 ; 95 \%$ confidence interval (CI) 0.52-0.97], a $43 \%$ reduction in symptomatic PE (0.7 vs. $1.2 \%$; HR $0.57 ; 95 \% \mathrm{CI} 18-60)$ and an overall reduction in symptomatic VTE of $36 \%$ (1.6 vs. $2.5 \%$; HR $0.64 ; 95 \%$ CI 19-50). There was a $58 \%$ reduction in fatal PE (0.27 vs. $0.64 \%$; HR $0.42 ; 95 \%$ CI $0.24-0.73)$. However, there was no difference in mortality up to day 35 in the overall HFS cohort (6.7 vs. $6.9 \%$; HR 0.97; $95 \%$ CI $0.85-1.1)$. In the elective arthroplasty cohort, there was no significant difference in VTE incidence between patients receiving aspirin and those receiving placebo (1.15 vs. $1.4 \%$; HR 0.81; $95 \%$ CI 0.47-1.42), nor was there any significant effect on mortality ( 9 vs. $11 \%$; HR $0.72 ; 95 \%$ CI 0.29-1.79). Overall, patients receiving aspirin had significantly more postoperative bleeds requiring transfusion compared to the placebo group ( 2.9 vs $2.4 \% ; p=0.04$ ).

The PEP investigators have been criticized for deemphasizing the primary outcomes specified in their original protocol (e.g., any vascular death, major non-fatal vascular events), choosing instead to focus on secondary outcomes of DVT, PE, or any VTE. They have also come under scrutiny for changes to sample size calculations and inadequate presentation of bleeding data $[44,45]$. The PEP trial, however, does possess some strengths, including randomization, blinding, large sample size, inclusion of only symptomatic events, and almost complete follow-up. While the PEP trial did show that aspirin is a viable alternative to no prophylaxis, it unfortunately did not address the question of whether or not aspirin is equivalent, non-inferior, or superior to anticoagulants for primary VTE prevention. Evidence suggests that LMWHs are better than aspirin for DVT prevention [46]. However, aspirin proponents are quick to point out the lack of direct evidence to show superiority of LMWH over aspirin in preventing the more concerning condition of $\mathrm{PE}$ or in reducing mortality [46].

\section{Could Alternative Primary VTE Prophylaxis Strategies} Be Adequate in Contemporary Practice?

Over the last two decades, major changes in surgical techniques, surgical populations, and post-procedural care plans may have impacted the risk of post-operative VTE. Prior to 1980 , the rate of symptomatic VTE among orthopedic surgery patients not receiving prophylaxis was approximately $15-30 \%$ [47-51]. A more recent observational study reported a much lower symptomatic VTE incidence of $2.7 \%$ (1.1 \% PE, $1.5 \%$ DVT, $0.6 \%$ both PE and DVT). This is likely attributable to shorter lengths of stay and use of multimodal VTE prophylaxis strategies that include pneumatic compression devices, earlier ambulation, and use of regional anesthesia [52]. In the most recent iteration of the ACCP guidelines (2012), baseline symptomatic VTE risk among major orthopedic surgery patients in the absence of prophylaxis is estimated to be $4.3 \%$ $(1.5 \%$ PE, $2.8 \%$ DVT [7•]). Even though the incidence appears to have decreased, VTE is potentially fatal, often clinically silent, and at-risk patients should be appropriately managed with evidence-based prophylactic regimens. Outcomes from older VTE prophylaxis studies may not be representative of the contemporary clinical situations. Given the reduced baseline risk of VTE, the net benefit of anticoagulant prophylaxis has likely changed. This issue is a key driving force behind rekindled interest of aspirin for primary VTE prophylaxis in recent years. Unfortunately, randomized controlled trials, retrospective reviews, and meta-analyses published since the PEP trial have been unable to consistently show an advantage for aspirin over anticoagulants in VTE reduction or bleeding among orthopedic surgery patients [53-63].

Two recent large retrospective analyses, based on data from the National Joint Registry (NJR) of England, compared LMWH with aspirin for primary VTE prevention among patients undergoing THR and TKR $[64,65]$. More than 265,000 patients between 2003 and 2008 were included and followed for 90 days. Roughly $20 \%$ of study patients received aspirin thromboprophylaxis and $80 \%$ received LMWH. The investigators reported no significant difference in the rates of VTE, 90-day mortality, or major hemorrhage (cerebrovascular or GI) between the two groups. Among TKR patients, there was a significantly greater risk of returning to the operating room within 
30 days among the aspirin patients as compared to the LMWH group [0.26 vs. $0.19 \%$; adjusted odds ratio (OR) 0.73 ; $95 \%$ CI $0.58-0.94]$. Among THR patients, a nonsignificant trend towards reduction in 90-day mortality in favor of LMWH was observed ( 0.61 vs. $0.65 \%$; adjusted OR $0.93 ; 95 \%$ CI $0.77-1.11 ; p=0.06)$. Limitations of these analyses include retrospective design, reliance on coding data, and potential for higher-risk patients preferentially receiving LMWH prophylaxis. The investigators suggested that, given the similar risk of VTE, 90-day mortality, and major hemorrhage between elective THR/ TKR patients receiving aspirin or LMWH, future VTE prophylaxis guidelines should consider incorporation of aspirin as "a viable alternative to LMWH" for VTE prevention.

In the recently published non-inferiority, multicenter, double-blind EPCAT trial [2••], patients undergoing THR were randomized to receive either aspirin $81 \mathrm{mg}$ daily for 28 days or dalteparin 5,000 units subcutaneously daily for 28 days after all patients had received an initial 10-day prophylactic course of dalteparin. The primary efficacy outcome was symptomatic proximal DVT or PE within 90 days of randomization. The primary safety outcome was major hemorrhage, defined as fatal bleeding, symptomatic bleeding into a critical area or organ, bleeding causing $\geq 20 \mathrm{~g} / \mathrm{l}$ decrease in hemoglobin, or requiring transfusion of $\geq 2$ units of blood. Secondary outcomes included death, clinically relevant non-major (CRNM) bleeding, myocardial infarction (MI), stroke, and wound infection. Investigators initially intended to recruit 1,100 patients per group. However, enrollment was stopped early due to commercial availability and significant provider uptake of rivaroxaban, which was approved for VTE prophylaxis during the study period. In an interim analysis by the Data
Safety and Monitoring Board (DSMB), it was determined that the endpoint of non-inferiority had been achieved after enrollment and randomization of 786 patients, and that further enrollment would not likely show a safety benefit for aspirin or an efficacy benefit for dalteparin. Adherence to study medications was self-reported as almost $93 \%$. Only 1 of the 380 aspirin patients had a VTE event compared to 5 of 398 in the dalteparin group in the 90-day follow period. Aspirin achieved non-inferiority, but not superiority, for prevention of VTE compared to dalteparin. There was no significant difference in major bleeding, CRNM bleeding, or any secondary outcomes, including death, MI, or stroke (Fig. 1).

In an analysis of net clinical benefit using the combined outcome of VTE, major bleeding and CRNM bleeding, a trend favoring aspirin was observed ( 0.8 vs. $2.5 \%$; absolute difference 1.7 percentage points; $95 \%$ CI $0.3-3.8$ percentage points; $p=0.091$; Fig. 1). This trial suggests that utilization of anticoagulants for the first 10 days postprocedure, combined with aspirin for an additional 28 days of extended prophylaxis, is a viable alternative to extended prophylaxis with anticoagulation in elective THR. A significant contribution to the literature by this study is the use of low-dose aspirin (81 mg daily) instead of higher-dose regimens often seen in VTE prophylaxis (e.g., $325 \mathrm{mg}$ twice daily). Evidence shows that aspirin doses beyond $65-100 \mathrm{mg}$ daily provide no further reduction in thromboembolic events, but do increase bleeding events [17]. The generalizability of the results from the EPCAT trial may be impacted by the availability of OMAs. As such, a second trial, EPCAT II, which compares aspirin to rivaroxaban for extended prophylaxis in THR patients, is underway. Results are anticipated in early 2018 (clinicaltrials.gov NCT01720108).
Fig. 1 Aspirin versus dalteparin for extended VTE prophylaxis after THR [2••], $* p$ not significant for superiority but significant for noninferiority $(p<0.001)$

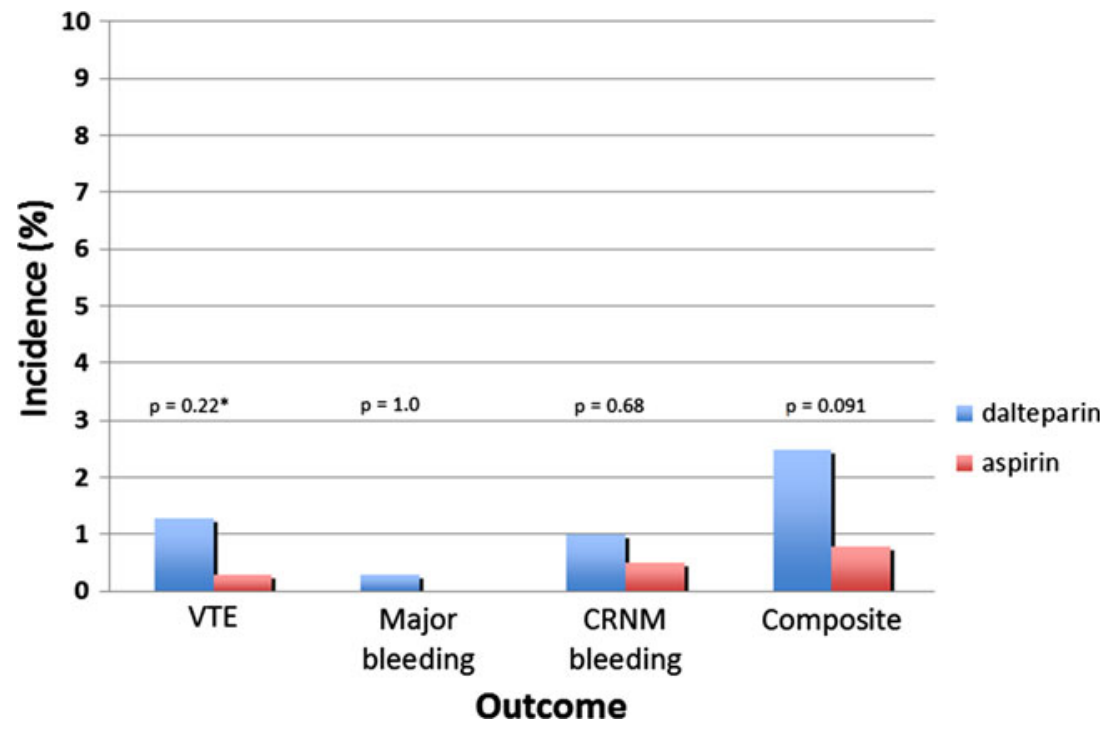




\section{Secondary Prevention of VTE After an Initial Anticoagulation Course}

Studies evaluating initial warfarin therapy ranging from 6 weeks to 12 months have found that rates of VTE recurrence remain at $15-20 \%$ within 2 years of discontinuation, regardless of the duration of warfarin therapy [66-69]. Two recent studies evaluating the use of aspirin in patients with a previous unprovoked VTE have suggested that aspirin may indeed have a role in secondary prophylaxis in patients who have discontinued or are intolerant to warfarin. The Aspirin to Prevent Recurrent Venous Thromboembolism (ASPIRE) and Warfarin and Aspirin (WARFASA) are sister trials that were designed to evaluate the efficacy and safety of aspirin in preventing recurrent VTE after an initial heparin/warfarin course in patients with an unprovoked, first episode of VTE $[3 \bullet \bullet, 4 \bullet \cdot$. In both trials, the primary efficacy outcome was recurrence of symptomatic VTE, and the primary safety outcome was fatal and nonfatal major bleeding. WARFASA evaluated patients with a duration of initial anticoagulation therapy of 6-18 months, while the ASPIRE trial evaluated patients with a duration of initial anticoagulation therapy of 6 weeks-24 months. After the initial anticoagulation course, both trials compared $100 \mathrm{mg}$ of aspirin to placebo for 2 years, with an option to extend treatment in WARFASA, or up to 4 years in ASPIRE.

The WARFASA trial found that recurrence of symptomatic VTE was significantly reduced by $42 \%$ with aspirin as compared to placebo (6.6 vs. $11.2 \%$ per year; HR $0.58 ; 95 \%$ CI 0.36-0.93). There was no difference in major bleeding, with only one non-fatal, major bleed in each group. There was no difference in rates of VTE recurrence between patients receiving an initial anticoagulation course lasting 6 months as compared to patients receiving a longer initial anticoagulation course. Due to slow recruitment in ASPIRE, the study was not sufficiently powered to detect the expected reduction in VTE events with aspirin. Therefore, during an interim analysis, investigators decided to prospectively synchronize their protocol and combine their results with the results of the WARFASA trial. When the ASPIRE results were examined alone, the trial failed to find a significant difference in recurrent VTE, although it did estimate a non-significant risk reduction of $26 \%$ in patients treated with aspirin. The ASPIRE did find significant reductions in the aspirin group in pre-defined secondary outcomes of major vascular events (VTE, MI, stroke, or cardiovascular death; 5.2 vs. $8 \%$ per year; HR $0.66 ; 95 \%$ CI $0.48-0.92 ; p=0.01)$. Net clinical benefit, defined as the reduction in the rate of composite of VTE, MI, stroke, major bleeding, or death from any cause was also significantly reduced with aspirin (6 vs. $9 \%$; HR $0.67 ; 95 \%$ CI $0.49-0.91$ ). When results were combined with those of the WARFASA trial, there was a significant reduction in VTE recurrence (13.8 vs. $19.1 \%$; HR $0.68 ; 95 \%$ CI $0.51-0.9 ; p=0.007$ ) and major vascular events (15.9 vs. $22.4 \%$; HR $0.66 ; 95 \%$ CI $0.51-0.86 ; p=0.002$ ) among patients receiving aspirin. In both studies and the pooled analysis, major bleeding was rare and rates were similar between groups.

\section{Suggested Future Research}

Although there has been a long-standing belief that aspirin does not play a significant role in VTE prevention, emerging data suggest it may be a reasonable option. However, further research is needed to validate the existing body of evidence. Specific areas of research that could help further define the role of aspirin include VTE prevention in orthopedic and nonorthopedic surgical patients, as well as non-surgical patients. Research should encompass well-designed, randomized clinical trials comparing aspirin against standards of care such as warfarin, OMAs, LMWH, and fondaparinux (both with and without an initial period of anticoagulant prophylaxis); aspirin against placebo or mechanical devices where ethical; and examining the optimal dose and duration of aspirin therapy for primary and secondary VTE prevention. Investigators should not discount the impact of advanced surgical techniques, a multimodal approach to VTE prophylaxis and aggressive post-operative care plans, which may have rendered previous clinical trial data somewhat obsolete. Study outcomes should ideally include mortality, although the large number of patients needed might make this unfeasible. Other outcomes may include quality of life, incidence of symptomatic VTE, non-VTE vascular events (e.g., MI, stroke), major hemorrhage, and net clinical benefit.

\section{Impact on Emergency and Hospitalist Physicians}

Use of aspirin for primary and secondary VTE prevention has the potential to affect the practice of emergency and hospitalist providers in several ways. Aspirin is available over the counter and may not always appear on the patient's formal prescription list within the electronic medical record. It will be important for providers to determine the exact indication for aspirin therapy (e.g., cardioprotection for coronary artery disease, primary or secondary VTE prevention, etc.) in a particular patient and clearly document this in the medical record. This will allow review of the medication profile for interactions that potentiate bleed risk, avoid critical errors of omission, and promote implementation of appropriate peri-procedural medication strategies.

Occasionally, a patient on warfarin for a prior VTE will present to the hospital and may have been maintained on anticoagulation beyond their initial prescribed, or intended, 
duration of therapy. Other patients may complete therapy during the hospitalization. Hospitalists and emergency providers may consider use of aspirin instead of anticoagulation in these patients to mitigate the risk of bleeding, simplify the patient's medication regimen, and possibly improve patient satisfaction and quality of life. If a shift from anticoagulation therapy to aspirin is made, it is imperative to counsel the patient and caregivers on the change and to encourage them to communicate this information to their outpatient providers as well as their retail pharmacist. Finally, use of aspirin for primary and secondary VTE prevention may shift medico-legal risk away from hospital providers as it would not require routine monitoring and adjustment, as does warfarin therapy. Emergency and hospitalist providers should stay abreast of emerging evidence regarding aspirin for VTE prevention and apply it to their practices accordingly.

\section{Conclusion}

Controversy has surrounded aspirin as a modality for primary and secondary prevention of VTE over the last several decades. It is possible, with major changes in surgical techniques and post-procedural care occurring in the last decade, that alternative prophylactic regimens are sufficiently effective in preventing VTE among orthopedic surgery patients. The ACCP and AAOS have both recently made significant changes to their VTE prevention guidelines, moving closer to a middle ground on the topic of aspirin. These changes seem to be somewhat prescient given the results of the EPCAT trial looking at aspirin for primary prevention, along with the WARFASA and ASPIRE trials, showing there may be a role for aspirin in secondary VTE prevention after an initial course of anticoagulation. National quality initiatives surrounding VTE prevention, such as SCIP, do not currently include aspirin as an appropriate sole antithrombotic option but will in the near future. Before aspirin can be recommended as a firstline agent or an alternative to anticoagulants for VTE prophylaxis, additional, well-designed studies are needed.

\section{Compliance with Ethics Guidelines}

Conflict of Interest Allison E. Burnett has served on the board for the 2013 Anticoagulation Forum and received honoraria for a presentation and for manuscript preparation, and was reimbursed for travel/accommodations expenses; has received compensation from Janssen Pharmaceutical and CSL Behring for service as a Consultant. Linda R. Kelly, Tuesdy Horner, Dukens LaBaze and Brandi N. Bowman declare that they have no conflict of interest. Charles E. Mahan has received compensation from Boehringer-Ingelheim, Sanofi, Leo Pharmaceuticals, Janssen, Bristol Myers Squibb, Eisai, and Polymedix for service as a Consultant. Charles E. Mahan is supported by Grants from Sanofi and the North American Thrombosis Forum; and has received honoraria from Boehringer-Ingelheim, Sanofi, Janssen, and Bristol Myers Squibb.

Human and Animal Rights and Informed Consent This article does not contain any studies with human or animal subjects performed by any of the authors.

\section{References}

Papers of importance, published recently, have been highlighted as:

- Of importance

•- Of major importance

1. Heit J, Cohen A, Anderson Jr F. Estimated annual number of incident and recurrent, non-fatal and fatal venous thromboembolism (VTE) events in the US. Blood (ASH Annu Meet Abstr). 2005;106(abstract \#910).

2. - Anderson DR, Dunbar MJ, Bohm ER, Belzile E, Kahn SR, Zukor D, et al. Aspirin versus low-molecular-weight heparin for extended venous thromboembolism prophylaxis after total hip arthroplasty: a randomized trial. Ann Intern Med. 2013;158(11): 800-6. This study is pivotal in that it is the first well-designed, randomized prospective trial to directly compare $L M W H$ versus aspirin for THR.

3. • Brighton TA, Eikelboom JW, Mann K, Mister R, Gallus A, Ockelford P, et al. Low-dose aspirin for preventing recurrent venous thromboembolism. N Engl J Med. 2012;367(21): 1979-87. This is the WARFASA trial that compares aspirin to placebo after an initial heparin/warfarin trial to assess rates of recurrent VTE.

4. • Becattini C, Agnelli G, Schenone A, Eichinger S, Bucherini E, Silingardi $\mathrm{M}$, et al. Aspirin for preventing the recurrence of venous thromboembolism. N Engl J Med. 2012;366(21): 1959-67. This is the ASPIRE trial that compares aspirin to placebo after an initial heparin/warfarin trial to assess rates of recurrent VTE. Pivotal design and important because recurrent VTE rates were significantly reduced in the primary trial alone.

5. Douketis JD, Spyropoulos AC, Spencer FA, Mayr M, Jaffer AK, Eckman $\mathrm{MH}$, et al. Perioperative management of antithrombotic therapy: antithrombotic therapy and prevention of thrombosis, 9th ed: American College of Chest Physicians evidence-based clinical practice guidelines. Chest. 2012;141(2 Suppl):e326S-50S.

6. Ageno W, Gallus AS, Wittkowsky A, Crowther M, Hylek EM, Palareti G, et al. Oral anticoagulant therapy: antithrombotic therapy and prevention of thrombosis, 9th ed: American College of Chest Physicians evidence-based clinical practice guidelines. Chest. 2012;141(2 Suppl):e44S-88S.

7. - Falck-Ytter Y, Francis CW, Johanson NA, Curley C, Dahl OE, Schulman S, et al. Prevention of VTE in orthopedic surgery patients: antithrombotic therapy and prevention of thrombosis, 9th ed: American College of Chest Physicians evidence-based clinical practice guidelines. Chest. 2012;141(2 Suppl):e278S325S. ACCP major orthopedic surgery guidelines.

8. Kearon C, Akl EA, Comerota AJ, Prandoni P, Bounameaux H, Goldhaber SZ, et al. Antithrombotic therapy for VTE disease: antithrombotic therapy and prevention of thrombosis, 9th ed: American College of Chest Physicians evidence-based clinical practice guidelines. Chest. 2012;141(2 Suppl):e419S-94S.

9. Hull RD, Pineo GF, Brant RF, Mah AF, Burke N, Dear R, et al. Long-term low-molecular-weight heparin versus usual care in proximal-vein thrombosis patients with cancer. Am J Med. 2006;119(12):1062-72. 
10. Lee AY, Levine MN, Baker RI, Bowden C, Kakkar AK, Prins M, et al. Low-molecular-weight heparin versus a coumarin for the prevention of recurrent venous thromboembolism in patients with cancer. N Engl J Med. 2003;349(2):146-53.

11. Garcia DA, Baglin TP, Weitz JI, Samama MM, American College of Chest Physicians. Parenteral anticoagulants: Antithrombotic therapy and prevention of thrombosis, 9th ed: American College of Chest Physicians evidence-based clinical practice guidelines. Chest. 2012;141(2 Suppl):e24S-43S.

12. Stewart DW, Freshour JE. Aspirin for the prophylaxis of venous thromboembolic events in orthopedic surgery patients: a comparison of the AAOS and ACCP guidelines with review of the evidence. Ann Pharmacother. 2013;47(1):63-74.

13. Milestones: the Bayer story 1863-1988 [Internet]. Leverkusen: Bayer AG; 1988. http://www.news.bayer.de/baynews/baynews. nsf/id/B695E754CDDDA468C1257AFD004ED5DE/\$file/Part_1_ Sites_1-169_Milestones.pdf. Updated Aug 1988; cited 2 May 2013.

14. Lansberg MG, O’Donnell MJ, Khatri P, Lang ES, Nguyen-Huynh MN, Schwartz NE, et al. Antithrombotic and thrombolytic therapy for ischemic stroke: antithrombotic therapy and prevention of thrombosis, 9th ed: American College of Chest Physicians evidence-based clinical practice guidelines. Chest. 2012;141(2 Suppl):e601S-36S.

15. Whitlock RP, Sun JC, Fremes SE, Rubens FD, Teoh KH, American College of Chest Physicians. Antithrombotic and thrombolytic therapy for valvular disease: antithrombotic therapy and prevention of thrombosis, 9th ed: American College of Chest Physicians evidence-based clinical practice guidelines. Chest. 2012;141(2 Suppl):e576S-600S.

16. You JJ, Singer DE, Howard PA, Lane DA, Eckman MH, Fang $\mathrm{MC}$, et al. Antithrombotic therapy for atrial fibrillation: antithrombotic therapy and prevention of thrombosis, 9th ed: American College of Chest Physicians evidence-based clinical practice guidelines. Chest. 2012;141(2 Suppl):e531S-75S.

17. Eikelboom JW, Hirsh J, Spencer FA, Baglin TP, Weitz JI. Antiplatelet drugs: antithrombotic therapy and prevention of thrombosis, 9th ed: American College of Chest Physicians evidence-based clinical practice guidelines. Chest. 2012;141(2 Suppl):e89S-119S.

18. Bhatt DL, Scheiman J, Abraham NS, Antman EM, Chan FK, Furberg CD, et al. ACCF/ACG/AHA 2008 expert consensus document on reducing the gastrointestinal risks of antiplatelet therapy and NSAID use: a report of the American College of Cardiology Foundation Task Force on Clinical Expert Consensus Documents. Circulation. 2008;118(18):1894-909.

19. O'Malley GF. Emergency department management of the salicylate-poisoned patient. Emerg Med Clin N Am. 2007;25(2): 333-46; abstract viii.

20. Li C, Hirsh J, Xie C, Johnston MA, Eikelboom JW. Reversal of the anti-platelet effects of aspirin and clopidogrel. J Thromb Haemost. 2012;10(4):521-8.

21. Monroe DM, Hoffman M. What does it take to make the perfect clot? Arterioscler Thromb Vasc Biol. 2006;26(1):41-8.

22. Paez Espinosa EV, Murad JP, Khasawneh FT. Aspirin: pharmacology and clinical applications. Thrombosis. 2012. doi:10.1155/ 2012/173124.

23. Kurtz S, Ong K, Lau E, Mowat F, Halpern M. Projections of primary and revision hip and knee arthroplasty in the united states from 2005 to 2030. J Bone Jt Surg Am. 2007;89(4):780-5.

24. Mahan CE, Kaatz S. Performance of new anticoagulants for thromboprophylaxis in patients undergoing hip and knee replacement surgery. Pharmacotherapy. 2012;32(11):1036-48.

25. Cardiovascular Disease Educational and Research Trust, Cyprus Cardiovascular Disease Educational and Research Trust, European Venous Forum, International Surgical Thrombosis Forum,
International Union of Angiology, Union Internationale de Phlebologie. Prevention and treatment of venous thromboembolism. International consensus statement (guidelines according to scientific evidence). Int Angiol. 2006;25(2):101-61.

26. Geerts WH, Bergqvist D, Pineo GF, Heit JA, Samama CM, Lassen MR, et al. Prevention of venous thromboembolism: American College of Chest Physicians evidence-based clinical practice guidelines (8th edition). Chest. 2008;133(6 Suppl):381S453S.

27. - Preventing venous thromboembolic disease in patients undergoing elective hip and knee arthroplasty [Internet]. Rosemont: American Academy of Orthopedic Surgeons; 2011. http://www. aaos.org/research/guidelines/VTE/VTE_guideline.asp. Updated 24 Sep 2011; cited 6 July 2013. AAOS major orthopedic surgery guidelines.

28. Cohen A. There's madness in their methods-a response to: venous thromboembolism prophylaxis: do trial results enable clinicians and patients to evaluate whether the benefits justify the risk? Proceedings of an ad hoc working group meeting. J Thromb Haemost. 2013;11(4):782-5.

29. Surgical care improvement project: percent of surgery patients with recommended VTE prophylaxis ordered anytime from hospital arrival to 24 hours after anesthesia end time [Internet]. Rockville: Agency for Healthcare Research Quality (AHRQ); 2012. http:// www.qualitymeasures.ahrq.gov/content.aspx?id=35537. Updated July 2012; cited 19 July 2013.

30. Cohen AT, Davidson BL, Gallus AS, Lassen MR, Prins MH, Tomkowski W, et al. Efficacy and safety of fondaparinux for the prevention of venous thromboembolism in older acute medical patients: randomised placebo controlled trial. BMJ. 2006; 332(7537):325-9.

31. Samama MM, Cohen AT, Darmon JY, Desjardins L, Eldor A, Janbon C, et al. A comparison of enoxaparin with placebo for the prevention of venous thromboembolism in acutely ill medical patients. Prophylaxis in medical patients with Enoxaparin Study Group. N Engl J Med. 1999;341(11):793-800.

32. Leizorovicz A, Cohen AT, Turpie AG, Olsson CG, Vaitkus PT, Goldhaber SZ, et al. Randomized, placebo-controlled trial of dalteparin for the prevention of venous thromboembolism in acutely ill medical patients. Circulation. 2004;110(7):874-9.

33. Collins R, Scrimgeour A, Yusuf S, Peto R. Reduction in fatal pulmonary embolism and venous thrombosis by perioperative administration of subcutaneous heparin. Overview of results of randomized trials in general, orthopedic, and urologic surgery. N Engl J Med. 1988;318(18):1162-73.

34. Turpie AG, Bauer KA, Eriksson BI, Lassen MR. Fondaparinux vs enoxaparin for the prevention of venous thromboembolism in major orthopedic surgery: a meta-analysis of 4 randomized double-blind studies. Arch Intern Med. 2002;162(16):1833-40.

35. Clagett GP, Reisch JS. Prevention of venous thromboembolism in general surgical patients. Results of meta-analysis. Ann Surg. 1988;208(2):227-40.

36. Antiplatelet Trialists' Collaboration. Collaborative overview of randomised trials of antiplatelet therapy-III: reduction in venous thrombosis and pulmonary embolism by antiplatelet prophylaxis among surgical and medical patients. BMJ. 1994;308(6923): 235-46.

37. Imperiale TF, Speroff T. A meta-analysis of methods to prevent venous thromboembolism following total hip replacement. JAMA. 1994;271(22):1780-5.

38. Gent M, Hirsh J, Ginsberg JS, Powers PJ, Levine MN, Geerts WH, et al. Low-molecular-weight heparinoid orgaran is more effective than aspirin in the prevention of venous thromboembolism after surgery for hip fracture. Circulation. 1996;93(1):80-4.

39. Gehling H, Giannadakis K, Lefering R, Hessmann M, Achenbach S, Gotzen L. Prospective randomized pilot study of ambulatory 
prevention of thromboembolism. 2 times $500 \mathrm{mg}$ aspirin (ASS) vs. clivarin 1750 (NMH). Unfallchirurg. 1998;101(1):42-9.

40. Lotke PA, Palevsky H, Keenan AM, Meranze S, Steinberg ME, Ecker ML, et al. Aspirin and warfarin for thromboembolic disease after total joint arthroplasty. Clin Orthop Relat Res. 1996;324:251-8.

41. Monreal M, Lafoz E, Roca J, Granero X, Soler J, Salazar X, et al. Platelet count, antiplatelet therapy and pulmonary embolism-a prospective study in patients with hip surgery. Thromb Haemost. 1995;73(3):380-5.

42. Powers PJ, Gent M, Jay RM, Julian DH, Turpie AG, Levine M, et al. A randomized trial of less intense postoperative warfarin or aspirin therapy in the prevention of venous thromboembolism after surgery for fractured hip. Arch Intern Med. 1989;149(4):771-4.

43. Prevention of pulmonary embolism and deep vein thrombosis with low dose aspirin: pulmonary embolism prevention (PEP) trial. Lancet. 2000;355(9212):1295-302.

44. Cohen A, Quinlan D. PEP trial. Pulmonary embolism prevention. Lancet. 2000;356(9225):247; author reply 250-1.

45. MacMahon S, Rodgers A, Collins R, Farrell B. Antiplatelet therapy to prevent thrombosis after hip fracture. Rationale for a randomised trial. J Bone Jt Surg Br. 1994;76(4):521-4.

46. Karthikeyan G, Eikelboom JW, Turpie AG, Hirsh J. Does acetyl salicylic acid (ASA) have a role in the prevention of venous thromboembolism? Br J Haematol. 2009;146(2):142-9.

47. Borgstroem S, Greitz T, van der Linden W, Molin J, Rudics I. Anticoagulant prophylaxis of venous thrombosis in patients with fractured neck of the femur; a controlled clinical trial using venous phlebography. Acta Chir Scand. 1965;129:500-8.

48. Eskeland G, Solheim K, Skjorten F. Anticoagulant prophylaxis, thromboembolism and mortality in elderly patients with hip fractures. A controlled clinical trial. Acta Chir Scand. 1966;131(1): $16-29$.

49. Galasko CS, Edwards DH, Fearn CB, Barber HM. The value of low dosage heparin for the prophylaxis of thromboembolism in patients with transcervical and intertrochanteric femoral fractures. Acta Orthop Scand. 1976;47(3):276-82.

50. Sautter RD, Koch EL, Myers WO, Ray JR III, Mazza JJ, Larson DE, et al. Aspirin-sulfinpyrazone in prophylaxis of deep venous thrombosis in total hip replacement. JAMA. 1983;250(19): 2649-54.

51. Sevitt S, Gallagher NG. Prevention of venous thrombosis and pulmonary embolism in injured patients. A trial of anticoagulant prophylaxis with phenindione in middle-aged and elderly patients with fractured necks of femur. Lancet. 1959;2(7110):981-9.

52. Bjornara BT, Gudmundsen TE, Dahl OE. Frequency and timing of clinical venous thromboembolism after major joint surgery. J Bone Jt Surg Br. 2006;88(3):386-91.

53. Brown GA. Venous thromboembolism prophylaxis after major orthopaedic surgery: a pooled analysis of randomized controlled trials. J Arthroplast. 2009;24(6 Suppl):77-83.

54. Bozic KJ, Vail TP, Pekow PS, Maselli JH, Lindenauer PK, Auerbach AD. Does aspirin have a role in venous thromboembolism prophylaxis in total knee arthroplasty patients? J Arthroplast. 2010;25(7):1053-60.

55. Brookenthal KR, Freedman KB, Lotke PA, Fitzgerald RH, Lonner JH. A meta-analysis of thromboembolic prophylaxis in total knee arthroplasty. J Arthroplast. 2001;16(3):293-300.

56. Freedman KB, Brookenthal KR, Fitzgerald RH Jr, Williams S, Lonner JH. A meta-analysis of thromboembolic prophylaxis following elective total hip arthroplasty. J Bone Jt Surg Am. 2000;82-A(7):929-38.
57. Westrich GH, Haas SB, Mosca P, Peterson M. Meta-analysis of thromboembolic prophylaxis after total knee arthroplasty. J Bone Jt Surg Br. 2000;82(6):795-800.

58. Gelfer Y, Tavor H, Oron A, Peer A, Halperin N, Robinson D. Deep vein thrombosis prevention in joint arthroplasties: continuous enhanced circulation therapy vs low molecular weight heparin. J Arthroplast. 2006;21(2):206-14.

59. Westrich GH, Bottner F, Windsor RE, Laskin RS, Haas SB, Sculco TP. VenaFlow plus lovenox vs VenaFlow plus aspirin for thromboembolic disease prophylaxis in total knee arthroplasty. J Arthroplast. 2006;21(6 Suppl 2):139-43.

60. Dorr LD, Gendelman V, Maheshwari AV, Boutary M, Wan Z, Long WT. Multimodal thromboprophylaxis for total hip and knee arthroplasty based on risk assessment. J Bone Jt Surg Am. 2007;89(12):2648-57.

61. Ji HM, Lee YK, Ha YC, Kim KC, Koo KH. Little impact of antiplatelet agents on venous thromboembolism after hip fracture surgery. J Korean Med Sci. 2011;26(12):1625-9.

62. Khatod M, Inacio MC, Bini SA, Paxton EW. Pulmonary embolism prophylaxis in more than 30,000 total knee arthroplasty patients: is there a best choice? J Arthroplast. 2012;27(2):167-72.

63. Intermountain Joint Replacement Center Writing Committee. A prospective comparison of warfarin to aspirin for thromboprophylaxis in total hip and total knee arthroplasty. J Arthroplast. 2012;27(1):1.e2-9.e2.

64. Jameson SS, Baker PN, Charman SC, Deehan DJ, Reed MR, Gregg PJ, et al. The effect of aspirin and low-molecular-weight heparin on venous thromboembolism after knee replacement: a non-randomised comparison using National Joint Registry data. J Bone Jt Surg Br. 2012;94(7):914-8.

65. Jameson SS, Charman SC, Gregg PJ, Reed MR, van der Meulen $\mathrm{JH}$. The effect of aspirin and low-molecular-weight heparin on venous thromboembolism after hip replacement: a non-randomised comparison from information in the National Joint Registry. J Bone Jt Surg Br. 2011;93(11):1465-70.

66. Pinede L, Ninet J, Duhaut P, Chabaud S, Demolombe-Rague S, Durieu I, et al. Comparison of 3 and 6 months of oral anticoagulant therapy after a first episode of proximal deep vein thrombosis or pulmonary embolism and comparison of 6 and 12 weeks of therapy after isolated calf deep vein thrombosis. Circulation. 2001;103(20):2453-60.

67. Kearon C, Gent M, Hirsh J, Weitz J, Kovacs MJ, Anderson DR, et al. A comparison of three months of anticoagulation with extended anticoagulation for a first episode of idiopathic venous thromboembolism. N Engl J Med. 1999;340(12):901-7.

68. Agnelli G, Prandoni P, Santamaria MG, Bagatella P, Iorio A, Bazzan M, et al.; Warfarin Optimal Duration Italian Trial Investigators. Three months versus one year of oral anticoagulant therapy for idiopathic deep venous thrombosis. N Engl J Med. 2001;345(3):165-9.

69. Schulman S, Rhedin AS, Lindmarker P, Carlsson A, Larfars G, Nicol P, et al. A comparison of six weeks with six months of oral anticoagulant therapy after a first episode of venous thromboembolism. Duration of Anticoagulation Trial Study Group. N Engl J Med. 1995;332(25):1661-5.

70. Holbrook A, Schulman S, Witt DM, Vandvik PO, Fish J, Kovacs MJ, et al. Evidence-based management of anticoagulant therapy: antithrombotic therapy and prevention of thrombosis, 9th ed: American College of Chest Physicians evidence-based clinical practice guidelines. Chest. 2012;141(2 Suppl):e152S-84S. 